\title{
II. The Quantum world
}

The motion of macroscopic objects such as soccer balls, vehicles and planets can be well described using classical mechanics. However, classical mechanics is inadequate to describe the movements or the structure of microscopic objects such as atoms, molecules or nuclei. To properly describe the properties of microscopic objects, the theory of quantum mechanics was developed. While quantum mechanics can be applied to macroscopic objects, it turns out that for objects of large dimensions the quantum mechanical laws evolve naturally into classical mechanics. In general, solving a problem in quantum mechanics is far more difficult than solving it in classical mechanics. Therefore, the laws of classical mechanics are employed when they can be applied safely.

As we descend to the nanoscale, the applicability of classical mechanics gradually diminishes. This does not occur at the same time for all of the variables describing the system, but below a certain size, quantum effects will become important. In this chapter, we briefly review some basic features of quantum mechanics. In the next chapter some of the consequences for nanotechnology of the appearance of quantum effects are discussed.

\section{Classical versus quantum mechanics}

The dynamics of motion of a classical object is characterized by the mass of the object, its position, its velocity (i. e., the speed and direction) or its momentum which is the product of the mass of the object times its velocity. The mass is fixed. The position and velocity or the momentum are continuous variables. For example, the speed of a car can vary continuously from zero to its maximum value. These variables characterizing the object can be measured as accurately as the tools used to make the measurement allow. The evolution of the position and velocity defines the trajectory of the car.

To describe transfers of energy not accompanied by transfers of matter, such as occurs when sound and light (or other electromagnetic radiations) move through space, classical physics employs a picture of wave propagation. The theoretical description is based on solving differential equations with boundary conditions depending upon the specific situation under consideration. For example, vibrations which are confined in space 
generate waves with definite frequencies. Thus, sound waves generated by vibration of a guitar string are periodic and depend on the nature of the string and its length. The string does not generate all sound frequencies (white noise) but just those satisfying the boundary conditions. If the vibration is not confined, as it is the case for waves propagating in free space, all frequencies are possible.

\section{Wave-particle duality}

At the macroscopic level, where classical physics applies, a particle always behaves as a particle and a wave always as a wave. In the microscopic quantum world particles and waves are not as easily separable. Depending upon the conditions, a particle can behave as a wave and a wave as a particle. For example, while, at the classical level light is described by wave equations, in the "photoelectric" and "Compton" effects, light behaves as a particle, the photon. This "wave-particle duality" has many practical implications. The photoelectric effect, ejection of electrons by collisions with photons, is exploited in photovoltaic cells, to transform sunlight into electricity. The Compton effect, scattering of photons by electrons, is used in imaging detectors for medical applications. Electrons or neutrons, which behave in many cases as particles, exhibit wave behavior evidenced by diffraction patterns analogous to those observed in the scattering of light and other electromagnetic radiations. Electron and neutron scattering are used to view small details of small objects and to determine the underlying structures of materials. These are just a few examples of the harnessing of quantum effects in devices that we use routinely. This behavior is schematically depicted in figures 12 and 13 .

The experimental observation of wave-particle duality led naturally to a description of microscopic matter in which it was postulated that each particle had associated with it a wave which governed its motion. The corresponding wavelength of this wave is known as the de Broglie wavelength.

\section{Determinism versus a probabilistic approach}

As indicated by the discussion so far, classical mechanics is a deterministic theory. If we know the initial conditions of the object (mass, position and speed) and the forces acting upon it (force field), it is possible to calculate 


\section{Classical effect}

\section{Quantum effect}
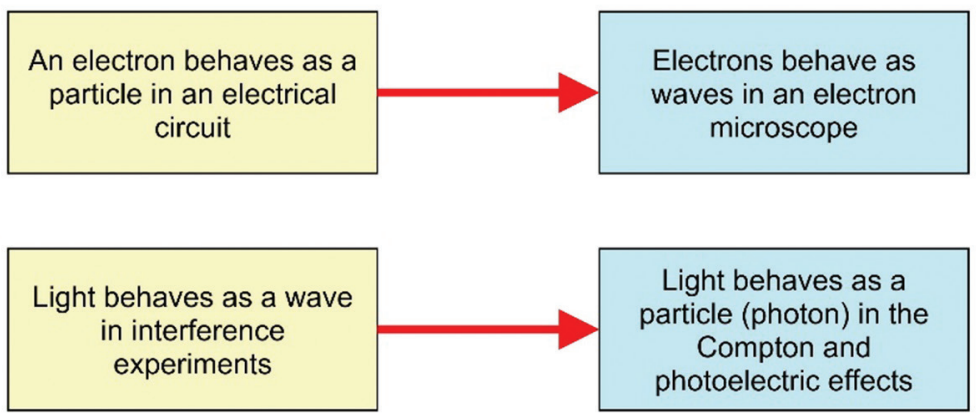

\section{Wave-particle duality}

Figure 12. Quantum mechanics accounts for the fact that a particle can behave as a wave or a wave as a particle according to the boundary conditions placed upon a system. This is illustrated for an electron and light (photons) in this figure.

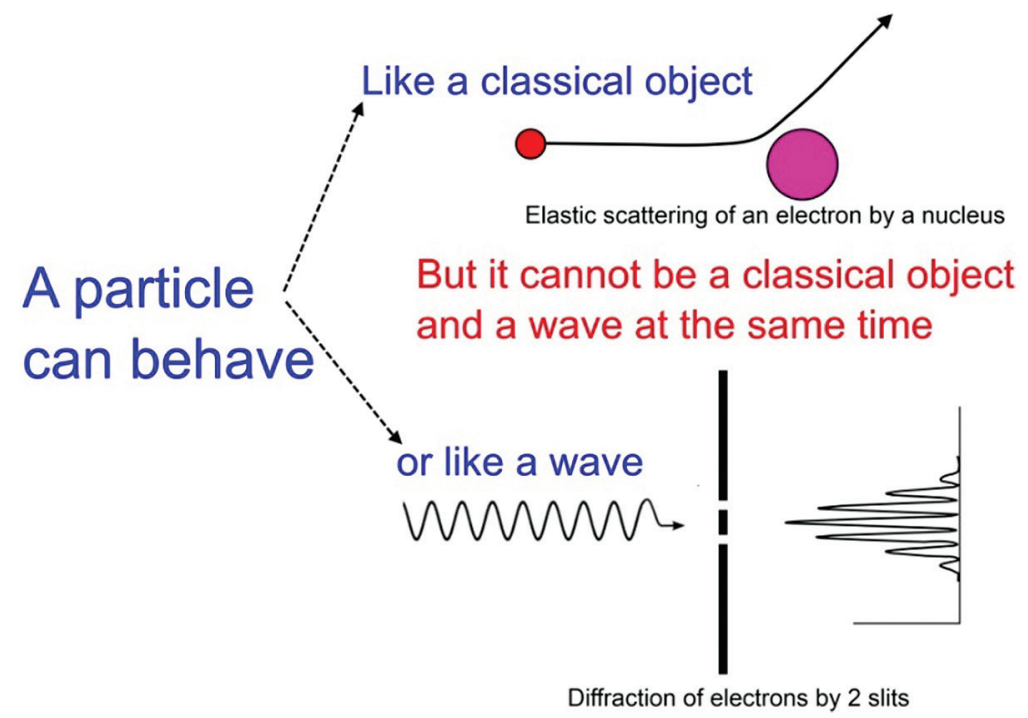

Figure 13. An electron can behave like a classical object or like a wave depending on the conditions, but it can never be both of them at the same time. This illustrates the wave-particle duality. 
the position and the velocity at any later time. The object follows a well-defined trajectory. It has been amply demonstrated experimentally that classical mechanics does not provide an appropriate description of microscopic entities such as atoms, molecules, electrons and nuclei. Instead, these particles manifest wave-particle duality which incorporates features of both particle and wave behaviors. Thus, classical mechanics is not adequate to treat microscopic entities. For these, Quantum Mechanics is the proper theory to apply.

In quantum mechanics the notion of trajectory does not exist anymore. The behavior of the quantum object cannot be characterized completely by its position and momentum, but rather requires a knowledge of its associated wave function. All of the information accessible for the system is contained within this wave function. The wave function is a solution to a wave equation, a differential operator equation which controls the motion of the particle. An operator equation is one in which some specified mathematical operation, e. g., multiplication, division, differentiation...., is performed on a given mathematical function. For each physical quantity that we can measure, there is an associated operator. The theory requires that such operators be "Hermitian" 2 . Figure 14 gives a schematic comparison of some aspects of classical and quantum mechanics.

\section{Measurement}

If we measure the speed of a car with radar waves, we can determine that speed with an error depending on the accuracy of the measuring device used. There is, in principle, no limit to the accuracy, except that of the device we employ. Quantum mechanics differs from classical mechanics in that a measurement is not always deterministic but probabilistic. In quantum mechanics, the result of a measurement can only be a value allowed by the wave function of the operator associated to the observable that we measure. Such an allowed value is called an "eigenvalue". If the system is initially in an "eigenstate", a state allowed by this operator, then the results will be deterministic: Our measurement will produce that particular eigenvalue. A second measurement of the same variable performed on the system will again give the same result.

If the system is initially not in an eigenstate corresponding to one particular eigenvalue of the observable, each of the possible eigenvalues corresponding to allowed states of the system can be, in principle, measured with a certain probability. The same measurement performed on a set of 


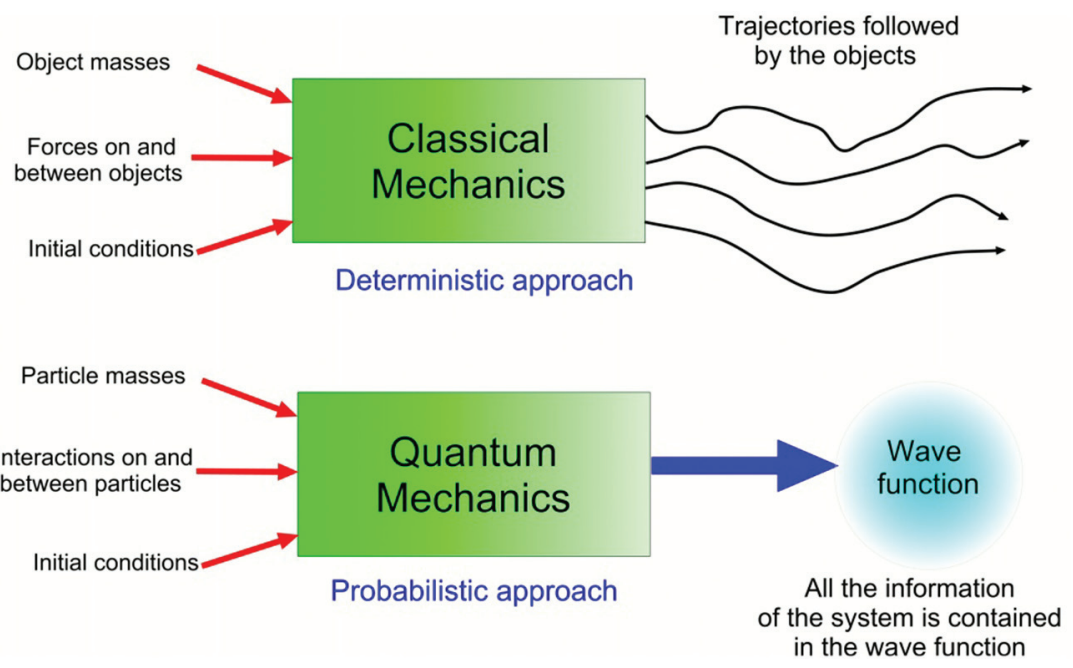

Figure 14. Comparison between classical and quantum mechanics.

such systems will give different results. Figure 15 summarizes this situation schematically.

\section{Quantization}

At the macroscopic level the position and the momentum of a particle are both continuous variables. The kinetic energy of a particle is also a continuous variable. The situation for particles exhibiting wave behavior is different. We have already recognized that confined waves, such as those produced by plucking a guitar string, generate one frequency and its harmonics, and not a white noise associated with continuous frequencies. This means that only certain discrete energy states of the system are allowed. This discretization of the possible energies comes from the boundary conditions which confine the string of the guitar on two sides. More generally stated, spatial boundary conditions lead to energy quantization.

As a result, particles confined (by external forces) to small regions of space cannot have continuous distributions in energy but can only have certain discrete energies. Further, it is interesting to note that, unlike the classical case, the lowest energy state of a quantum system cannot be at zero energy. Even in the lowest energy "ground" state, the position of the particle fluctuates. The non-zero value of the energy is called the "zero point energy". 


\section{Quantum system: illustration of a measurement}

Measurement of the variable $\mathbf{A}=>$ operator $\hat{\mathbf{A}}$
Eigenvalues of $\hat{\mathbf{A}}: \mathbf{a}_{1}, \mathrm{a}_{2}, \mathrm{a}_{3}, \ldots$
Eigenvectors (wave function) of $\hat{\mathbf{A}}: \Psi_{\mathrm{a} 1}, \Psi_{\mathrm{a} 2}, \Psi_{\mathrm{a} 3}, \ldots$

\begin{tabular}{|c|c|c|}
\hline $\begin{array}{c}\text { The system is initially } \\
\text { in state } \Psi_{\mathrm{a} 3}\end{array}$ & $\underset{\substack{\text { Measurement } \\
\text { of } A}}{\longrightarrow} a_{3}$ & $\underset{\substack{\text { Measurement } \\
\text { of } A}}{\longrightarrow} a_{3}$ \\
\hline
\end{tabular}

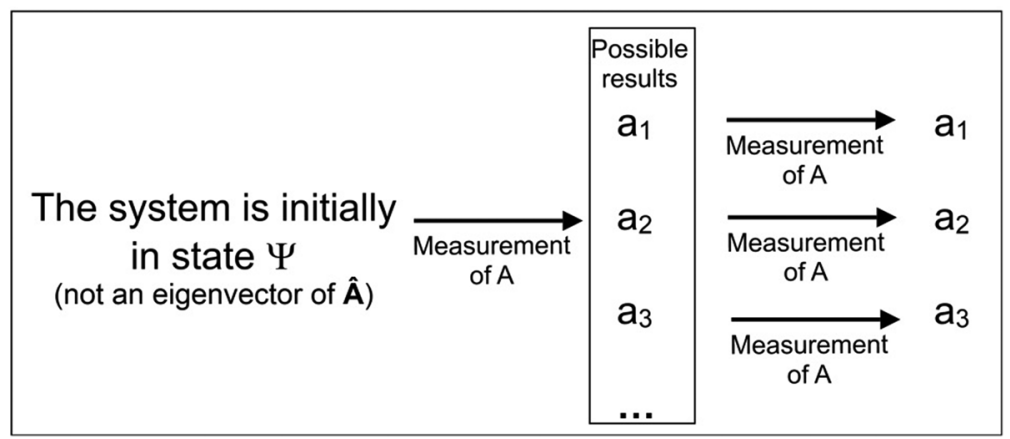

Figure 15. Illustration of results of a measurement on a quantum system. The variable (observable $A$ ) is measured and $\hat{A}$ is the associated operator. If the wave function is in an eigenstate of $\hat{A}$, for example $\psi_{\text {aз }}$, one measures a value $a_{3}$. A second measurement will give the same result. If the system is not in an eigenstate of $\hat{A}$, all the eigenvalues are possible, but with different probabilities.

The simple schematic picture in Figure 16 illustrates these concepts. The external forces confining the particle are represented by a parabola (harmonic oscillator potential). In the classical case shown on the left, the particle can be at any distance above the bottom of this parabola and have any energy up to the maximum energy $E$ indicated by the dashed lines. On the right, in the quantum case, only certain discrete energy levels of the system are allowed. These are indicated by horizontal solid lines. In this illustration, only the lower three states are allowed to the system. The energy spacing between the states will increase if the region in which the particle is confined decreases.

\section{Heisenberg uncertainty principle}

In principle, it is always possible to make a measurement on a classical system without notably perturbing it. Measuring the speed of a car with radar waves does not materially modify the trajectory of the car. Thus, it is possible to measure the position and the momentum (or velocity) 


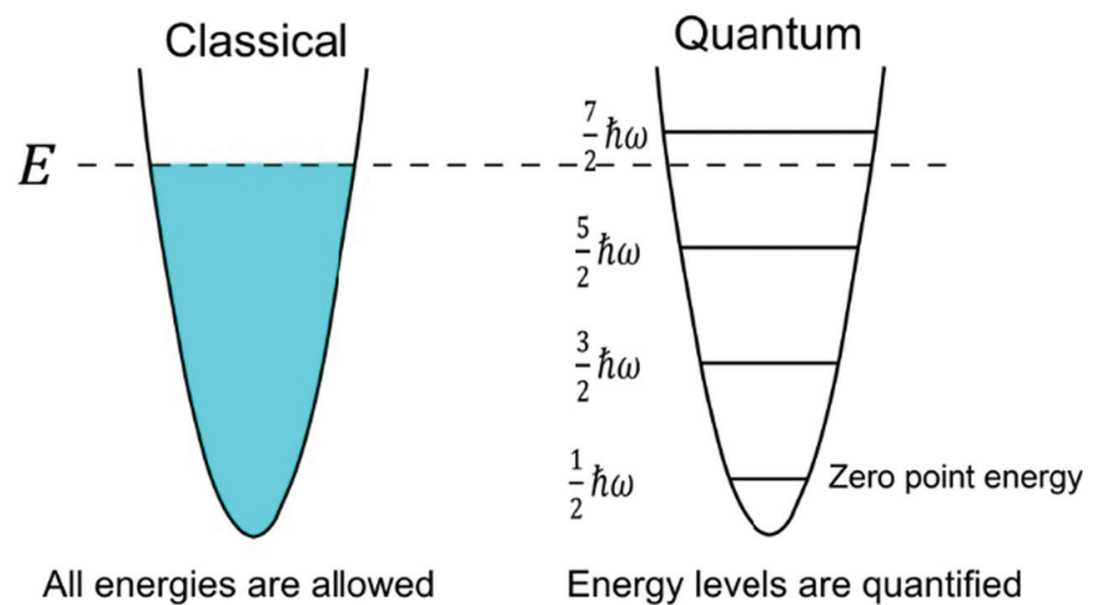

Figure 16. Particle confined by external forces. For the classical case on the left, all the energies from the bottom up to $\mathrm{E}$ are allowed (colored in the figure). For a quantum system only discrete energy values are allowed. Note that in the quantum case the lowest energy is greater than zero.

simultaneously. The accuracy of the measurement depends on the accuracy of the measuring instrument.

At the microscopic level the situation is different. In quantum mechanics, an operator is associated with each variable which can be measured and the result of a measurement is necessarily an "eigenvalue" of the operator. In contrast to the classical case, an accurate simultaneous measurement of two variables (having two different operators) is not possible unless the system is in an "eigenstate" common for both operators. This is related to the "commutivity" or "non-commutivity" of the two operators ${ }^{4}$.

If the two operators do not commute ${ }^{5}$, it is impossible to measure them simultaneously with all the accuracy we wish. This is true of position and momentum which are non-commuting variables. If, for a particle, $\Delta \mathrm{X}$ and $\Delta \mathrm{P}_{\mathrm{x}}$ are, respectively, the uncertainties in the position and momentum along the $\mathrm{x}$ axis, then the "Heisenberg uncertainty principle" may be stated as:

$$
\Delta \mathrm{X} \times \Delta \mathrm{P}_{\mathrm{x}} \geqslant \hbar
$$

Where $\hbar$ is the Planck constant divided by $2 \pi(\hbar=h / 2 \pi)$. It is possible to measure either the position or the momentum as accurately as we want but not both simultaneously. The accuracies achievable are determined by the equation above. A small uncertainty in one of these variables leads to a large uncertainty in the other. 
Figure 17 illustrates this correlation of uncertainties in the $\mathrm{x}$ and $\mathrm{p}_{\mathrm{x}}$ determinations, reflecting the Heisenberg uncertainty principle. The colored rectangles indicate the accuracy limits resulting from the non-commutivity. This same limitation also explains the zero point energy indicated in figure 16. Since the system is confined in a limited portion of space there will be an associated uncertainty in the momentum, and, as a consequence, on the energy. Neither the momentum or the energy can be zero. Associated with this zero-point energy is a zero-point motion. The particle cannot be at rest.

\section{Quantum numbers}

A system is completely defined by the knowledge of its wave function. In the case where the energy levels are discrete, the wave function often depends on indices which characterize a particular energy state of the system. We can designate the wave function of the system, $\psi_{n}$. Here $n$ is an integer number which can be o (the lowest energy or "ground" state) or a positive integer. If $n=1$, the system is in the first excited state, $n=2$, the second excited state, etc. Then, each state can be identified by the appropriate integer, which is known as a quantum number. The energy of the state will depend upon this quantum number. For a system with $N$ degrees of freedom there will be $N$ quantum numbers. (The number of degrees of freedom is the number of parameters of a system which can vary independently.) A classical system with $N$ degrees of freedom requires $N$ position coordinates. For a point particle having no internal degrees of freedom this number is equal to 3 (the three coordinates locating the position). Similarly, a quantum system with $N$ degrees of freedom has $N$ quantum numbers to describe it completely. For example, a point particle inside a cubic box has 3 degrees of freedom and the energy levels depend on 3 quantum numbers.

\section{Spin - an intrinsic property}

A particle can also have intrinsic degrees of freedom which do not depend on spatial position. One such intrinsic degree of freedom for particles such as electrons, protons, neutrons, nuclei etc. is known as angular momentum or spin. Sometimes the spin of a particle is represented as a rotation of the particle around an axis passing through its center. The spin is then a vector. This classical view helps to imagine what a spin can be. Spin is also quantized in quantum systems. Usually quantum angular momenta are measured in 


\section{Heisenberg uncertainty principle}
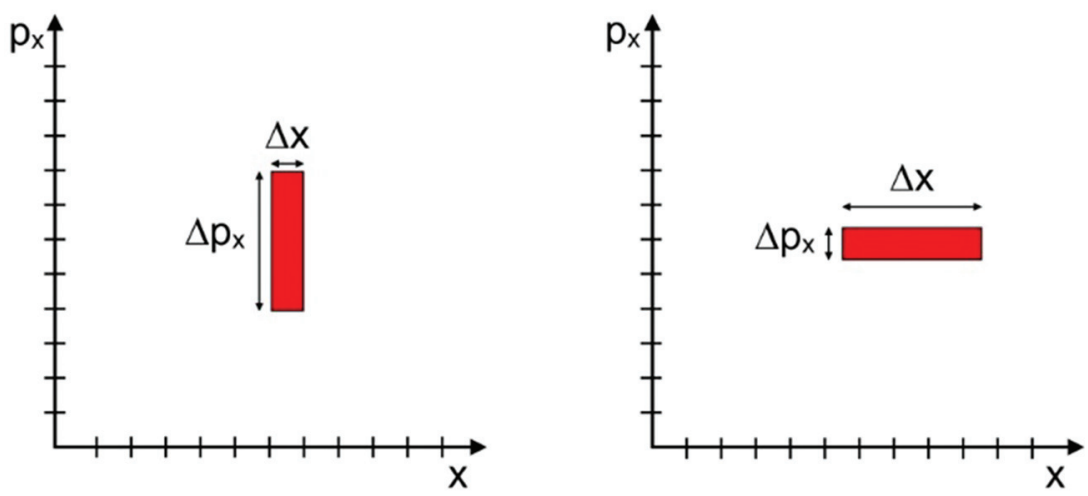

Figure 17. Illustration of the Heisenberg uncertainty principle for the position $(x)$ and the momentum (px) of a particle. These two quantities cannot be measured simultaneously. The better one of these variables is known, the greater is the uncertainty on the other.

$\hbar$ units. Electrons or protons, for example, have a spin $S=1 / 2 \hbar$ and we say that the spin of an electron or a proton is $s=1 / 2$. Quantum mechanics also says that the projection of this $1 / 2 \hbar$ vector on a given axis can take only two values: $+1 / 2 \hbar$ and $-1 / 2 \hbar$, often referred to as up or down. For larger spins, whether half-integer or integer, more projections (actually $2 \mathrm{~s}+1$ values) are possible.

All of the quantum particles found in nature have a spin. This spin can be either an integral spin $(0,1,2 \ldots)$, or a half-integral spin $(1 / 2,3 / 2,5 / 2 \ldots)$. Particles with integral spins are called bosons and particles with half-integral spins are called fermions.

\section{Fermions and bosons}

At the microscopic level, identical particles are indistinguishable. There is no way to differentiate between two electrons, for example. This is in severe contrast to our experience with macroscopic objects such as billiard balls which are, or can be made to be, discernible, e. $g$., by painting them different colors.

Symmetry plays a major role in nature and in quantum mechanics. The application of quantum mechanics to a set of identical particles requires that the total wave function is either totally symmetric with respect to the exchange of any two identical particles, i. e., the sign of the wave function 
does not change when the particles are exchanged, or totally antisymmetric with respect to the exchange of two identical particles, i.e., the sign of the wave function changes when the particles are exchanged. This symmetry requirement is intimately connected to the intrinsic spin of the particles. For bosons, the wave function is symmetric while for fermions it is antisymmetric. Bosons are said to obey Bose-Einstein statistics and fermions to obey Fermi-Dirac statistics.

One of the consequences of this is that, while it is possible to put as many identical bosons as we want into a given quantum state as in the right part of figure 18, completely identical fermions cannot occupy the same quantum state (a quantum state is one identified by all of the pertinent quantum numbers). In the left part in figure 18 we see that 2 fermions of identical spin $1 / 2$ can be placed in a single energy level. This comes from the fact that there are two possibilities for the spin projection, $\mathrm{s}_{\mathrm{z}}= \pm 1 / 2$. The two particles with these different spin projections are not completely identical. However, given the limitation of two spin projections, a third particle of spin $1 / 2$ cannot be placed in the same energy level. In the absence of an external magnetic field the spin of a particle is randomly oriented and the energies for different spin orientations are the same. The application of an external magnetic field results in a splitting of the energy levels as the spins will be oriented only in certain allowed directions relative to the magnetic field.

\section{Quantum tunneling}

Classical energy waves, e.g. electromagnetic waves or sound waves, can tunnel through a potential barrier and an attenuated wave can reach the other side (consider for example the attenuation of a sound wave passing through an acoustic tile). This situation is illustrated in the top of figure 19. There, a classical wave incident from the left impinges on the barrier. In the forbidden region an evanescent wave is formed with a decaying intensity and a wave of diminished amplitude emerges from the other side of the barrier.

In contrast, if a classical particle is incident on such a barrier and its kinetic energy is below the maximum energy of the barrier, it cannot pass through the barrier. To do so requires an energy at least equal to the barrier energy. A tennis ball does not tunnel through a concrete wall because it behaves as a classical object. Classical wave and particle behaviors are different. 


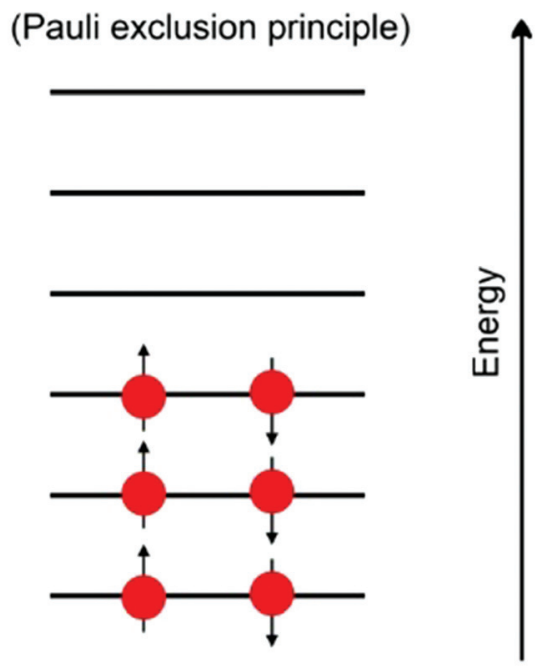

Fermions

\section{Fermion of spin $1 / 2$}

(Bose condensation)

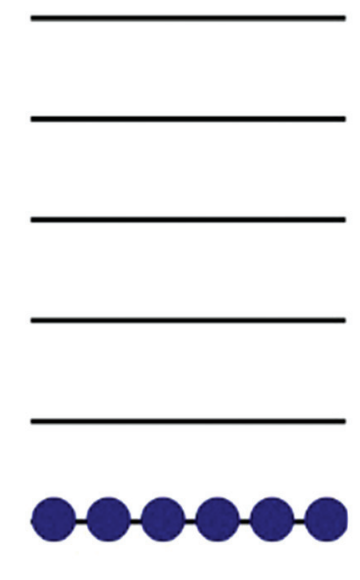

\section{Bosons}

Boson of spin 0

Figure 18. There are two families of particles at the quantum level: fermions and bosons. Completely identical fermions cannot be in the same quantum state. In the case of $s=1 / 2$ particles illustrated here, two different projections of the spin are allowed so two otherwise identical fermions can occupy a single energy level. In contrast, the number of identical bosons in the same quantum state is not restricted.

In a quantum mechanical treatment, wave-particle duality means that in certain conditions a particle can behave as a wave and exhibit a similar tunneling behavior. That is, there is a certain probability that the particle will pass through the barrier and be found on the other side. This is illustrated in the bottom part in figure 19. This behavior is indeed observed for particles such as electrons, protons, neutrons, nuclei, etc. This is precisely the phenomenon used in the scanning tunneling microscope to see and move atoms on a surface.

As it is schematically illustrated in figure 20 , the probability that a particle is able to tunnel through the barrier depends on the relationship between the particle energy and the height of the barrier. It is easier to tunnel with energies close to the top of the barrier than with smaller kinetic energies. The width of the barrier is also important. The larger the width, the smaller will be the tunneling probability. 

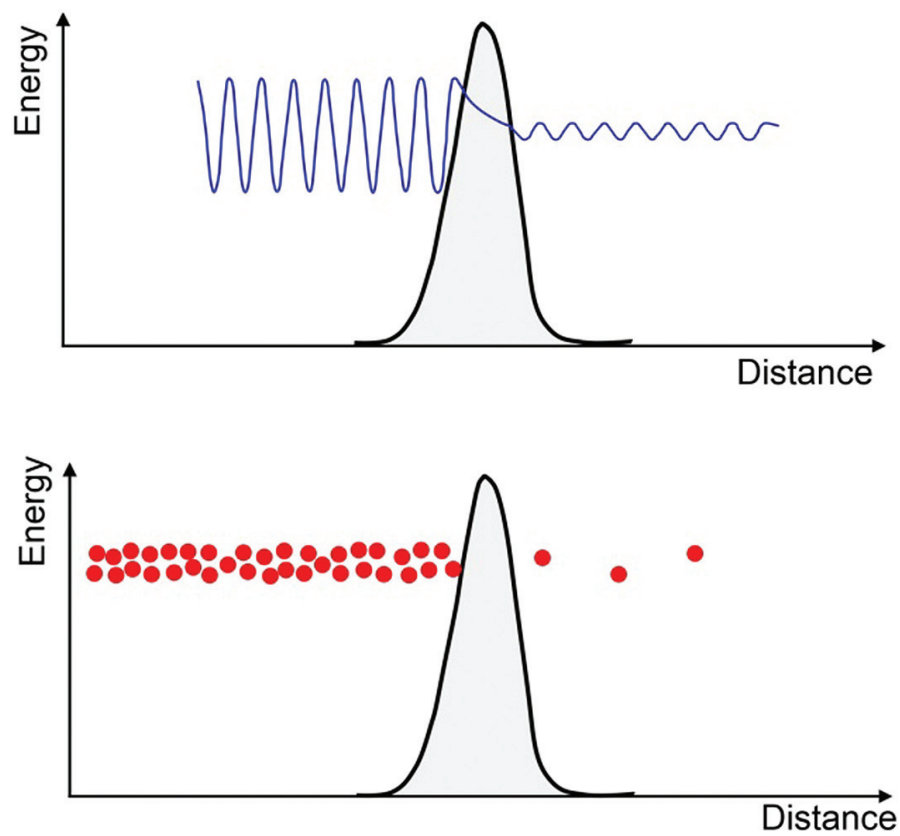

\section{Quantum tunneling through a barrier}

Figure 19. Schematic illustration of quantum tunneling. Top: the wave function decays exponentially inside the barrier and has a lower amplitude after passing through the potential barrier. Bottom: a small fraction of particles striking the barrier tunnel through the barrier.

\section{Summary}

A given system has a behavior which is governed by the laws of physics. By looking at different observables of a system, we can characterize the system. Observables are measureable variables that describe the properties of the system. Observables might be energies, positions, angular momenta, etc. Classical physics (mechanics, hydrodynamics, optics...) describes our macroscopic world well. Classical physics allows us to precisely calculate the trajectories of planets, rockets, vehicles, etc. The microscopic world is very different from the classical world. To adequately describe this microscopic world requires that we apply the concepts of quantum mechanics. This is not because the underlying scientific laws are different. It is rather because the boundary conditions have changed and the relative importance of certain exigencies of the scientific laws can become more or less important with respect to each other depending on the size of the piece of matter we are dealing with. Some effects, which are negligible at the 


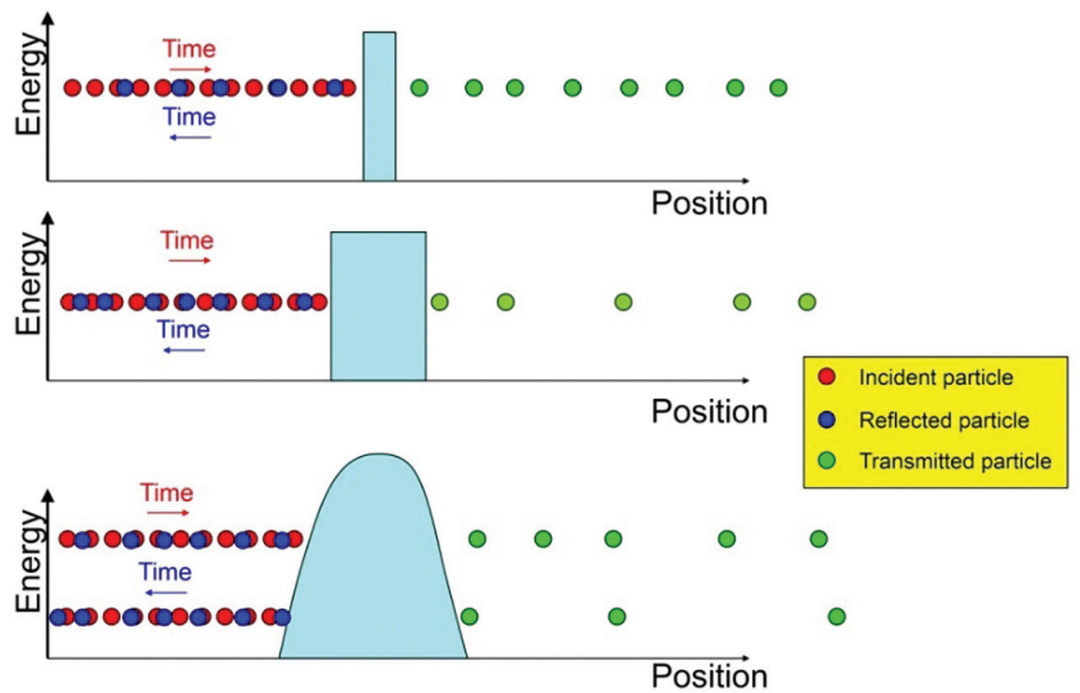

Figure 20. This figure illustrates, in a very simplified and schematic way, the fact that the probability to tunnel through a potential barrier decreases as the difference between the particle energy and the energy at the top of the barrier or the width of the barrier increase. In the figure red particles represent the incident flux on the barrier. Part of this incident flux tunnels through the barrier (green particles) while the remaining part bounces back (blue particles).

macroscale level, can become dominant at the microscale level. Although quantum mechanics is known to provide the foundation for accurately describing microscopic systems and predicting their behavior, a number of the concepts introduced in this chapter can be disconcerting when first encountered. 